\title{
A Cinesioterapia no tratamento de incontinência urinária em idosas: Revisão
}

\section{narrativa}

\author{
Kinesiotherapy in the treatment of urinary incontinence in the elderly: Narrative review \\ Cinesioterapia em el tratamiento de la incontinencia urinaria en ancianos: Revisión narrativa
}

Recebido: 21/12/2021 | Revisado: 28/12/2021 | Aceito: 15/01/2022 | Publicado: 17/01/2022

Thauan Narciso de Lima Ferro

ORCID: https://orcid.org/0000-0002-0174-6467 Centro Universitário Mario Pontes Jucá, Brasil

E-mail: thauan.ferro@umj.edu.br

Maria Aparecida da Silva

ORCID: https://orcid.org/0000-0003-2052-8008

Centro Universitário Mario Pontes Jucá, Brasil

E-mail: mariaparecida20161@outlook.com.br

\begin{abstract}
Resumo
A cinesioterapia é um conjunto de exercícios terapêuticos que ajudam na reabilitação de diversas situações, fortalecendo e alongando os músculos, e também podem servir para otimizar o estado de saúde geral e prevenir alterações motoras. Uma das doenças tratadas pela cinesioterapia é a incontinência urinária que se dá pela perda involuntária da urina pela uretra. O distúrbio é mais frequente no sexo feminino e pode manifestar-se tanto na quinta ou sexta década de vida quanto em mulheres mais jovens. O presente estudo teve como objetivo descrever A cinesioterapia no tratamento de incontinência urinária em idosas, por meio de uma revisão narrativa. As buscas foram realizadas na base de dados Scielo, BVS, Google acadêmico. A busca inicial resultou em 26 artigos e, ao final, foram selecionados 20 artigos para compor essa revisão. A presente revisão encontrou benefícios da cinesioterapia como a melhora ou cura da perda de urina, prevenção de incontinência urinária, reeducação e fortalecimento da musculatura pélvica. Constatou-se que A cinesioterapia no tratamento de incontinência urinária em idosas se faz importante, pois atua com exercícios para fortalecimento da musculatura pélvica é o reforço da resistência uretral e a melhora dos elementos de sustentação dos órgãos pélvicos.
\end{abstract}

Palavras-chave: Cinesioterapia em idosos; Incontinência urinária em idosos; Cinesioterapia em incontinência; Exercício de Kegel; Fisioterapia na incontinência; Assoalho pélvico.

\begin{abstract}
Kinesiotherapy is a set of therapeutic exercises that help in the rehabilitation of different situations, strengthening and stretching the muscles, and can also serve to optimize the general state of health and prevent motor alterations. One of the diseases treated by kinesiotherapy is urinary incontinence, which is caused by the involuntary loss of urine through the urethra. The disorder is more frequent in females and can manifest itself in the fifth or sixth decade of life as well as in younger women. The present study aimed to describe Kinesiotherapy in the treatment of urinary incontinence in elderly women, through a narrative review. Searches were performed in the Scielo, BVS, Google academic database. The initial search resulted in 26 articles and, in the end, 20 articles were selected to compose this review. The present review found benefits of kinesiotherapy such as improving or curing urine loss, preventing urinary incontinence, reeducating and strengthening the pelvic muscles. It was found that Kinesiotherapy in the treatment of urinary incontinence in elderly women is important, as it works with exercises to strengthen the pelvic muscles, it reinforces urethral resistance and improves the supporting elements of the pelvic organs.
\end{abstract}

Keywords: Kinesiotherapy in the elderly; Urinary incontinence in the elderly; Kinesiotherapy in incontinence; Kegel exercise; Physical therapy; Incontinence and pelvic floor.

\section{Resumen}

La kinesioterapia es un conjunto de ejercicios terapéuticos que ayudan en la rehabilitación de diferentes situaciones, fortaleciendo y estirando la musculatura, pudiendo también servir para optimizar el estado general de salud y prevenir alteraciones motoras. Una de las enfermedades tratadas por la kinesioterapia es la incontinencia urinaria, que es causada por la pérdida involuntaria de orina a través de la uretra. El trastorno es más frecuente en mujeres y puede manifestarse en la quinta o sexta década de la vida, así como en mujeres más jóvenes. Este estudio tuvo como objetivo describir la Kinesioterapia en el tratamiento de la incontinencia urinaria en mujeres mayores, a través de una revisión narrativa. Las búsquedas se realizaron en la base de datos académica de Scielo, BVS, Google. La búsqueda inicial resultó en 26 artículos y, al final, se seleccionaron 20 artículos para componer esta revisión. La presente revisión encontró beneficios de la kinesioterapia como mejorar o curar la pérdida de orina, prevenir la incontinencia urinaria, reeducar y fortalecer 
los músculos pélvicos. Se encontró que la Kinesioterapia en el tratamiento de la incontinencia urinaria en mujeres mayores es importante, ya que trabaja con ejercicios para fortalecer la musculatura pélvica, refuerza la resistencia uretral y mejora los elementos de soporte de los órganos pélvicos.

Palabras clave: Kinesioterapia en ancianos; Incontinencia urinaria en ancianos; Kinesioterapia en incontinencia; Ejercicio de Kegel; Fisioterapia en incontinencia; Suelo pélvico.

\section{Introdução}

De acordo com o IBGE (2018), o número da população idosa no Brasil aumentou $18 \%$ nos últimos cinco anos, o que torna necessário uma atenção maior à saúde das pessoas idosas. Com a ampliação da expectativa de vida, estima-se que a prevalência de incontinência urinária-IU também irá aumentar. Mundialmente, cerca de 348 milhões de pessoas apresentaram sintomas de incontinência urinaria. (Paiva et al., 2019).

O processo de envelhecimento, embora fisiológico, é permeado por maior vulnerabilidade às doenças, que podem interferir, entre outros, na autonomia, mobilidade, destreza manual, lucidez e capacidade funcional das vias urinárias inferiores e da bexiga, favorecendo a IU. (Langoni et al., 2014).

A idade é um fator de risco para a IU, devido às alterações hormonais que a mulher sofre no climatério, em que ocorre a redução do nível de estrógeno causando a diminuição da capacidade vesical e a perda de integridade de tônus da musculatura lisa e estriada, das fáscias e ligamentos que sustentam a bexiga e a uretra na sua posição ideal. Essas alterações decorrentes do processo de envelhecimento e os eventos de crises de saúde podem favorecer o desenvolvimento de incontinência urinária na velhice. O processo de envelhecimento como fenômeno isolado não é causa, mas induz a alterações anatômicas e funcionais que predispõem ao problema. (Krinski et al., 2013).

A incontinência urinária é a perda de urina em quantidade e frequência suficientes para causar desconforto ao indivíduo. No Brasil, a prevalência de IU entre idosos residentes em área urbana varia de $17 \%$ a 30,5\% para homens e de 31,1\% a 69,5\% entre as mulheres. (Cesar et al., 2018).

A Incontinência Urinária é classificada pela Sociedade Internacional de Incontinência (ICS) como Incontinência Urinária de Esforço (IUE), Incontinência Urinária de Urgência (IUU), Incontinência Urinária Mista (IUM), Incontinência Funcional, Incontinência Postural, Incontinência Insensível e Incontinência Coital. As três primeiras são as que mais ocorrem, sendo que a IUE é a forma mais comum (50-70\% dos casos) caracterizada por ocorrer após exercício físico, espirro ou tosse; a IUU se apresenta por forte desejo de urinar; e a IUM quando é seguido por apresentar a incontinência de urgência de esforço (Rodrigues et al., 2016; Topuz \& Sevig, 2016).

A fisioterapia, como forma abrangente de tratamento, visa a prevenção e tratamento curativo da IU através da educação da função miccional, informação a respeito do uso adequado da musculatura do assoalho pélvico, bem como o aprendizado de técnicas e exercícios para aquisição do fortalecimento muscular. São objetivos principais da fisioterapia a reeducação da musculatura do assoalho pélvico e seu fortalecimento, visto que, na maioria dos tipos de incontinência urinária, está presente uma redução da força desta musculatura. A cinesioterapia do assoalho pélvico compreende basicamente na realização dos exercícios de Kegel, que objetiva trabalhar a musculatura perineal para o tratamento da hipotonia do assoalho pélvico. (Oliveira et al., 2011).

Essa pesquisa justifica-se pela importância de entender qual o papel do fisioterapeuta no atendimento de idosas com incontinência urinária levando em consideração a cinesioterapia como tratamento.

\section{Metodologia}

Trata-se de um estudo de revisão narrativa que é uma metodologia de pesquisa, que busca estabelecer possíveis respostas, ou aproximar de respostas, a partir de questões mais amplas. As etapas para fazer uma revisão narrativa são: 1- Leitura. 
Selecionar os trabalhos de relevância sobre o assunto Proposto. 2- Resumo. Montar pequenos resumos sobre cada trabalho escolhido. 3-Resultados. Apresentar os resultados obtidos a partir da leitura e resumo dos trabalhos. As bases de dados utilizadas para a busca de artigos foram a Scientific Electronic Library Online (SciELO), google acadêmico e a Biblioteca Virtual em Saúde do Ministério da Saúde (BVS). Foram selecionados apenas artigos originais publicados nos últimos 10 anos. A pesquisa foi mediada pelos Descritores em Ciências da Saúde (DeCS)" "Cinesioterapia em idosos“", "Incontinência urinária em idosos", "Cinesioterapia em incontinência", "exercício de kegel”, "fisioterapia na incontinência" e " assoalho pélvico "Como critérios de inclusão foram selecionados artigos originais completos, que tratavam da cinesioterapia como tratamento da incontinência urinaria em idosas. Por fim foram excluídos artigos fora do tema e com período de publicação diferente do proposto no trabalho.

\section{Resultados}

Para sistematizar o resultado foi criado o Quadro 1 quantificando os artigos encontrados por base de dados.

Quadro 1 - Acervo em cada base de dados científicos selecionados.

\begin{tabular}{|l|c|c|c|}
\hline \multicolumn{1}{|c|}{ Base de dados } & $\begin{array}{c}\text { Total de artigos } \\
\text { encontrados }\end{array}$ & $\begin{array}{c}\text { Total de artigos } \\
\text { excluídos }\end{array}$ & Total de artigos selecionados \\
\hline Scielo & 9 & 3 & 6 \\
\hline Google acadêmico & 10 & 2 & 8 \\
\hline BVS & 7 & 1 & 6 \\
\hline Total & $\mathbf{2 6}$ & $\mathbf{6}$ & $\mathbf{2 0}$ \\
\hline
\end{tabular}

Fonte: Elaboração dos autores (2021).

Após separados por base de dados, foi organizado o resultado bibliográfico no Quadro 2 para organizar os achados em ordem cronológica. 
Quadro 2 - Síntese de resultados sobre a utilização de cinesioterapia na incontinência urinária de idosas.

\begin{tabular}{|c|c|c|}
\hline Autor & $\begin{array}{r}\text { Título } \\
\end{array}$ & Ano \\
\hline Jesus, E. S. \& Cartaxo, E. & $\begin{array}{l}\text { A fisioterapia na incontinência urinária da } \\
\text { mulher idosa: revisão de literatura. }\end{array}$ & 2008 \\
\hline Oliveira, J.R et al. & $\begin{array}{l}\text { Cinesioterapia no tratamento da } \\
\text { Incontinência Urinária em mulheres idosas. }\end{array}$ & 2011 \\
\hline Pereira, V. S et al. & $\begin{array}{l}\text { Efeitos do tratamento fisioterapêutico em } \\
\text { mulheres idosas com incontinência urinária: } \\
\text { uma revisão sistemática. }\end{array}$ & 2011 \\
\hline Rosa, L. H. T. et al & $\begin{array}{l}\text { Prevalência da incontinência urinária em } \\
\text { idosos de porto alegre - RS. }\end{array}$ & 2012 \\
\hline Krinski, G.G et al & $\begin{array}{l}\text { Os benefícios do tratamento fisioterapêutico } \\
\text { na incontinência urinária de esforço em } \\
\text { idosas - revisão sistemática. }\end{array}$ & 2013 \\
\hline Previatto, A.B. et al & $\begin{array}{l}\text { Atuação da fisioterapia na incontinência } \\
\text { urinária em mulheres idosas. }\end{array}$ & 2013 \\
\hline Langoni, C.S. et al & $\begin{array}{l}\text { Incontinência urinária em idosas de Porto } \\
\text { Alegre: sua prevalência e sua relação com a } \\
\text { função muscular do assoalho pélvico. }\end{array}$ & 2013 \\
\hline Guerra, T. E C. et al. & $\begin{array}{l}\text { Atuação da fisioterapia no tratamento de } \\
\text { incontinência urinaria de esforço. }\end{array}$ & 2014 \\
\hline Silva, R. E.G. et al & $\begin{array}{l}\text { Tratamento cinesioterapêutico como medida } \\
\text { de prevenção da incontinência urinária de } \\
\text { esforço em mulheres idosas e a sua relação } \\
\text { com a qualidade de vida. }\end{array}$ & 2015 \\
\hline Marques, S.R & $\begin{array}{l}\text { Tratamento fisioterapêutico na incontinência } \\
\text { urinária em idosas. }\end{array}$ & 2016 \\
\hline Rosa, T.S. M. et al & $\begin{array}{l}\text { Risco de quedas em idosos } \text { com } \\
\text { incontinência: uma revisão integrativa. }\end{array}$ & 2016 \\
\hline Cestari, C. E. et al. & $\begin{array}{llll}\text { Impacto da incontinência urinária na } \\
\text { qualidade de vida de idosas. }\end{array}$ & 2017 \\
\hline Rodrigues, T.S & $\begin{array}{l}\text { Atuação da fisioterapia na incontinência } \\
\text { urinária em idosas. }\end{array}$ & 2018 \\
\hline Cesar, J.A. et al & $\begin{array}{l}\text { Incontinência urinária entre idosos: um } \\
\text { estudo em áreas pobres do norte e nordeste } \\
\text { do Brasil. }\end{array}$ & 2018 \\
\hline Pires, M.N. et al & $\begin{array}{l}\text { Intervenção de Fisioterapia no tratamento de } \\
\text { incontinência urinária em idosas através do } \\
\text { método de kegel: Uma Revisão sistemática. }\end{array}$ & 2018 \\
\hline Souza, J. O. et al & $\begin{array}{l}\text { Influência da fisioterapia na qualidade de } \\
\text { vida de mulheres com incontinência urinária } \\
\text { por esforço. }\end{array}$ & 2019 \\
\hline Paiva, L.L. et al & $\begin{array}{l}\text { Prevalência de incontinência urinária em } \\
\text { idosos no brasil nos últimos } 10 \text { anos: uma } \\
\text { revisão sistemática. }\end{array}$ & 2019 \\
\hline Silveira, C. M. et al & $\begin{array}{l}\text { Os efeitos dos Exercícios de Kegel em idosas } \\
\text { com Incontinência Urinária: uma Revisão } \\
\text { Sistemática. }\end{array}$ & 2019 \\
\hline Funatsue $\mathrm{A} . \mathrm{H}$ et al & $\begin{array}{l}\text { A fisioterapia no tratamento da incontinência } \\
\text { urinária em mulheres idosas: uma revisão de } \\
\text { literatura. }\end{array}$ & 2020 \\
\hline Almeida, L. K.O. et al & $\begin{array}{l}\text { Fisioterapia no tratamento de incontinência } \\
\text { urinária em mulheres idosas: uma revisão } \\
\text { integrativa. }\end{array}$ & 2020 \\
\hline
\end{tabular}

Fonte: Elaboração dos autores (2021).

\section{Discussão}

O processo de envelhecimento e suas consequências naturais, as alterações biomorfológicas, são preocupações da humanidade desde o início da civilização. Naturalmente os seres vivos são regidos por um determinismo biológico: todos nascem, crescem, amadurecem, envelhecem, declinam e morrem. As alterações inerentes ao envelhecimento dependem de cada indivíduo, 
da programação genética de sua espécie e de fatores ambientais (habitat, modos de vida, e de agressões que tenham sofrido no decorrer de suas existências) (Pires et al., 2018).

Do número total de idosos, no Brasil, metade é representado por pessoas do sexo feminino. No ano 2000, para cada 100 mulheres idosas, havia 81 homens idosos. Em 2050, é provável que haverá cerca de 76 idosos para 100 idosas. (Rosa et al., 2012).

Pela sua elevada ocorrência, por demandar ampla utilização de serviços de saúde e pelos diversos transtornos trazidos ao indivíduo e à família, a incontinência urinária é um importante problema de saúde entre idosos. Vale destacar ainda que até 2060, a população idosa brasileira deverá quadruplicar, passando de 15 milhões para cerca de 58,4 milhões. (Cesar et al., 2018).

De maneira equivocada muitas mulheres acreditam que a perda de urina é algo normal do envelhecimento, ocorrendo também devido ao sentimento de vergonha e pelo desconhecimento da existência de tratamento para minimização ou cura dos sintomas, desta forma não há procura do acompanhamento profissional adequado (Almeida et al., 2020).

São observados prejuízos em mulheres com incontinência urinária quanto à atividade profissional, relações sociais, familiares e diminuição da atividade sexual das mulheres incontinentes. Além das consequências descritas acima, ainda são descritos traumas psicológicos relacionados à incontinência urinária, levando a sentimentos de humilhação, ansiedade, solidão e culpa. Essas mulheres cultivam o medo de serem ridicularizadas e consideradas idosas e inúteis. (Silva et al., 2015).

Literaturas mostram que a IU interfere na vida das pessoas de maneira física, ocupacional, socioeconômica, psicológica e sexual, afetando de forma significativa a qualidade de vida (Souza et al., 2019 apud Knorts et al., 2013).

$\mathrm{O}$ comprometimento do desempenho neuromuscular, evidenciado pela lentidão dos movimentos, pela perda de força e fadiga muscular precoce, constitui um aspecto marcante do envelhecimento dos seres humanos, gerando um círculo vicioso caraterizado por diminuição da qualidade de vida e alterações funcionais (Cestári et al., 2017).

Apesar da prevalência dessa patologia em várias as faixas etárias, dados comprovam que com o aumento da idade (mais de 65 anos) a probabilidade de possuir algum grau de IU é de 8 a 35\%. Em geral, cerca de 11\% das mulheres brasileiras sofrem com esse tipo de doença procurando, assim, algum atendimento clínico (Oliveira et al., 2011).

Os fatores de risco da IU são multifatoriais. O estudo epidemiológico permitiu identificar os fatores de risco que alguns autores sintetizam: fatores intrínsecos; fatores obstétricos e ginecológicos. (Rodrigues, 2018 apud Mascarenhas, 2011).

A incontinência urinária é a perda de urina em quantidade e frequência suficientes para causar desconforto ao indivíduo. No Brasil, a prevalência de IU entre idosos residentes em área urbana varia de 17\% a 30,5\% para homens e de 31,1\% a 69,5\% entre as mulheres (Cesar et al., 2018).

A IU é classificada de acordo com os sintomas apresentados, os três tipos mais encontrados são: incontinência urinária de esforço (IUE) - quando há perda da urina pelo meato uretral sincrônica com a realização de esforços urgeincontinência (UI) - caracterizada pela perda urinária seguida da urgência miccional; incontinência urinária mista (IUM) - ocorre perda urinária tanto à urgência quanto na realização de esforços. É uma das mais importantes e frequentes síndromes geriátricas sendo um problema comum que pode comprometer pessoas de todas as idades, contudo sua prevalência é maior na população feminina e aumenta com o avanço da idade (Krinski et al., 2013).

Pode-se considerar que os indivíduos com IU apresentam outras alterações musculoesqueléticas associadas, o que pode aumentar o risco de quedas nesta população, que podem ser definidas como o deslocamento não intencional do corpo para um nível inferior à posição inicial com incapacidade de correção de tempo hábil, determinado por circunstâncias multifatoriais, comprometendo a estabilidade (Rosa et al. apud Pereira et al., 2001).

O assoalho pélvico possui músculos que são classificados como músculos estriados esqueléticos, possuindo contração voluntária. Esses músculos exerce uma função contra a força gravitacional e a pressão intra-abdominal. Além disso, permite o suporte das vísceras pélvicas e do abdômen auxiliando de forma satisfatória na continência urinária e fecal. É valido ressaltar 
que uma diminuição da força dessa musculatura causa vários problemas, como por exemplo, a incontinência urinária. (Silveira et al. apud Alperin et al., 2014).

O planejamento das ações em saúde se apoia no uso de dados epidemiológicos para o direcionamento das suas estratégias e ações. Através do seu uso é possível desenvolver medidas que podem levar à prevenção das principais causas etiológicas de uma determinada doença ou disfunção e, com isso, impactar positivamente na saúde e na qualidade de vida da população, assim como delinear um tratamento mais efetivo e adequado para a população estudada. (Langoni et al., 2013).

A avaliação fisioterapêutica esclarecerá as situações que desencadeiam as perdas de urina; como por exemplo: tossir, espirrar, levantar-se, caminhar, manter relações sexuais, entre outras, pois o tipo de incontinência é classificado de acordo com a sintomatologia apresentada pela paciente. (Previatto et al., 2013).

Os testes para avaliar o assoalho pélvico são: a eletromiografia, o estudo histomorfológico por biópsia muscular ou avaliação clínica pela palpação bidigital vaginal, perineômetro e cones vaginais. A palpação bidigital vaginal é o método mais simples, por não requerer instrumentação e pode fornecer dados quantitativos da força e da resistência muscular durante a contração da musculatura do assoalho pélvico da paciente. O perineômetro é um equipamento pneumático que se baseia no mesmo princípio do esfigmotensiômetro, o que é constituído de uma sonda vaginal que infla, conectada a um manômetro onde indica ao paciente e ao terapeuta a intensidade da pressão exercida durante a contração (Marques apud Zanetti et al., 2009).

O tratamento fisioterapêutico tem como objetivo a melhora ou a cura da perda da urina, visando a prevenção por meio da educação da função miccional, informação a respeito do uso adequado da musculatura do assoalho pélvico, bem como o aprendizado de técnicas e exercícios para aquisição do fortalecimento muscular, sendo objetivo principal da fisioterapia a reeducação da musculatura do assoalho pélvico e seu fortalecimento (Previatto et al., 2013).

O tratamento conservador é realizado através de técnicas que objetivam o fortalecimento da musculatura do assoalho pélvico, pois a disfunção muscular constitui importante fator causal de perdas urinárias (Jesus et al., 2008 apud Herrmann et al., 2003).

A cinesioterapia do assoalho pélvico compreende basicamente na realização dos exercícios de Kegel, que objetiva trabalhar a musculatura perineal para o tratamento da hipotonia do assoalho pélvico. Kegel e Powel foram os primeiros pesquisadores nos Estados Unidos a prescrever exercícios específicos para o fortalecimento dos músculos do assoalho pélvico. O objetivo básico dos exercícios para fortalecimento da musculatura pélvica é o reforço da resistência uretral e a melhora dos elementos de sustentação dos órgãos pélvicos. (Oliveira et al., 2011).

Os programas de exercícios devem ter uma prescrição apropriada e que resulte em melhora da função muscular. Os exercícios utilizados como tratamento baseiam-se na hipótese de que os músculos se adaptam as sobrecargas a que são submetidos. Desta forma, para que ocorra um aumento na força, este músculo deve ser requisitado repetidamente contra uma resistência cada vez maior, sem produzir trauma (Guerra et al., 2014).

Dentre tudo, as orientações são necessárias, onde inclui-se mudanças de hábitos alimentares, como a diminuição da ingestão de cafeinados, excesso de líquidos antes de dormir, frutas ácidas, achocolatados e refrigerantes. Esses são considerados irritantes vesicais e por isso podem agravar os episódios de perdas urinárias, portanto tais mudanças devem ser estimuladas (Funatsu et al., 2020).

O acompanhamento em longo prazo dos resultados do tratamento fisioterapêutico é essencial para as conclusões sobre a efetividade da intervenção realizada. Dentre os estudos selecionados que realizaram esse acompanhamento, todos envolviam o fortalecimento da musculatura do assoalho pélvico. Sabe-se que, para a manutenção dos benefícios do fortalecimento da musculatura do assoalho pélvico, é necessária a continuação dos exercícios. (Pereira et al., 2011). 
Considerando o envelhecimento geral da população e o fato de a idade ser um fator de risco para a IU, um entendimento quanto à prevalência dessa disfunção em idosos é importante para a criação de estratégias terapêuticas direcionadas para este grupo da população. (Paiva et al., 2018).

\section{Conclusão}

Pode-se concluir que a cinesioterapia no tratamento da incontinência urinária é positiva para obter melhoras reais e significativas, atuando na prevenção e reabilitação da musculatura do assoalho pélvico, no alívio dos sinais e sintomas referidos, bem como na qualidade de vida das idosas portadoras de IU. Devido á alta prevalência dessa doença que se faz mais recorrente no sexo feminino.

A cinesioterapia tem benefícios nos principais tipos de incontinência urinária que são: IU de esforço, relacionada à escape urinário em situações de esforço (com Graus leves a moderados); IU de urgência, relacionada à vontade intensa e não controlável de urinar e IU mista que apresenta sinais e características dos dois tipos citados, promovendo o fortalecimento, alongamento e propriocepção da musculatura do assoalho pélvico.

Em suma, a fisioterapia tem demonstrado bastante eficiência na saúde da mulher com ênfase no tratamento cinesioterapeutico no tratamento da incontinência urinária. Com tudo se faz necessário estudos futuros sobre a implementação da cinesioterapia nas diversas fases da vida da mulher com finalidade de prevenir e tratar as incontinências urinárias.

\section{Referências}

Almeida, L. K. O. et al. (2020). Fisioterapia no tratamento de incontinência urinária em mulheres idosas: uma revisão integrativa. Anais do VII CIEH... Realize Editora. https://editorarealize.com.br/artigo/visualizar/73503.

Cesar, J. A. et al. (2018). Incontinência urinária entre idosos: um estudo em áreas pobres do norte e nordeste do brasil. Revista Baiana de Saúde Pública. 42(2), $231-243$.

Cestari, C. E. et al. (2017). Impacto da incontinência urinária na qualidade de vida de idosas. Revista Ciência E Estudos Acadêmicos De Medicina. 1(07), 2737. https://periodicos.unemat.br/index.php/revistamedicina/article/view/1773.

Funatsu, A. H. et al. (2020). A fisioterapia no tratamento da incontinência urinária em mulheres idosas: uma revisão de literatura. Centro Universitário Católico Salesiano Auxilium -UniSALESIANO de Araçatuba - SP. https://fisiosale.com.br/wp/wp-content/uploads/2019/02/A-fisioterapia-no-tratamento-daincontin\%C3\%AAncia-urin\%C3\%A1ria-em-mulheres-idosas-uma-revis\%C3\%A3o-de-literatura.pdf

Guerra, T. E C. et al. (2014). Atuação da fisioterapia no tratamento de incontinência urinaria de esforço. Femina. 42(6), 251-254. http://files.bvs.br/upload/S/0100-7254/2014/v42n6/a4823.pdf.

Jesus, E. S. \& Cartaxo, E. (2008). A fisioterapia na incontinência urinária da mulher idosa: revisão de literatura. Universidade Católica do Salvador. http://ri.ucsal.br:8080/jspui/handle/prefix/3730

Krinski, G. G. et al. (2013). Os benefícios do tratamento fisioterapêutico na incontinência urinária de esforço em idosas - revisão sistemática. Brazilian Journal of Surgery and Clinical Research - BJSCR. 4(3), 37-40.

Langoni, C. S. et al. (2013). Incontinência urinária em idosas de Porto Alegre: sua prevalência e sua relação com a função muscular do assoalho pélvico. FAENFI/PUCRS - Porto Alegre (RS).

Marques, S. R. (2016). Tratamento fisioterapêutico na incontinência urinária em idosas. Revista saúde integrada. 9(17).

Oliveira, J. R. et al. (2011). Cinesioterapia no tratamento da Incontinência Urinária em mulheres idosas. rev. bras. geriatr. Gerontol, 14(2), 343-351.

Paiva, L. L. et al. (2019). Prevalência de incontinência urinária em idosos no brasil nos últimos10 anos: uma revisão sistemática. Estud. interdiscipl. envelhec. $24,275-293$.

Pereira, V. S. et al. (2012). Efeitos do tratamento fisioterapêutico em mulheres idosas com incontinência urinária: uma revisão sistemática. Rev Bras Fisioter, São Carlos. 16(6), 463-8.

Pires, M. N. et al. (2013). Intervenção de Fisioterapia no tratamento de incontinência urinária em idosas através do método de kegel: Uma Revisão sistemático. Pós-graduação em Geriatria e Gerontologia - Faculdade Ávila.

Previatto, A. B. et al. (2013). Atuação da fisioterapia na incontinência urinária em mulheres idosas. Centro Universitário Católico Salesiano Auxilium- Araçatuba - Sp. 
Research, Society and Development, v. 11, n. 2, e1111225023, 2022

(CC BY 4.0) | ISSN 2525-3409 | DOI: http://dx.doi.org/10.33448/rsd-v11i2.25023

Rodrigues, M. P. et al. (2016). Perfil das pacientes do ambulatório de uroginecologia de um Hospital Público de Porto Alegre com relação à incontinência urinária e à qualidade de vida. Revista Clin. Biomed Res. 36, 135-141. https://doi.org/10.25248/reas.e734.2019

Rodrigues, T. S. (2018). Atuação da fisioterapia na incontinência urinária em idosas. FAEMA -Ariquemes/RO. http://repositorio.faema.edu.br:8000/jspui/handle/123456789/2429.

Rosa, L. H. T. et al. (2012). Prevalência da incontinência urinária em idosos de porto alegre - RS. Revista geriatria \& gerontologia.

Rosa, T. S. M. et al. (2016). Risco de quedas em idosos com incontinência: uma revisão integrativa. Revista Kairós Gerontologia. 19(1), 161-173.

Silva, R. E. G. et al. (2015). Tratamento cinesioterapêutico como medida de prevenção da incontinência urinária de esforço em mulheres idosas e a sua relação com a qualidade de vida. Journal of Amazon Health Science. 1(1).

Silveira, C. M. et al. (2019). Os efeitos dos Exercícios de Kegel em idosas com Incontinência Urinária: uma Revisão Sistemática. REAS/EJCH. 26 (e734).

Souza, J. O. et al. (2019). Influência da fisioterapia na qualidade de vida de mulheres com incontinência urinária por esforço. Revista Científica do UBM. 21(40), 167-183. https://doi.org/10.52397/rcubm.v21i40.941 